\title{
Differential diagnoses and management strategies in patients with schizophrenia and bipolar disorder
}

\author{
A Carlo Altamura' \\ Jose M Goikolea ${ }^{2}$ \\ 'Department of Psychiatry, University \\ of Milan, IRCCS Fondazione Ospedale \\ Maggiore Policlinico Mangiagalli e \\ Regina Elena, Milan, Italy; ${ }^{2}$ Bipolar \\ Disorder Program, Hospital Clinic i \\ Universitari, Barcelona, Spain
}

\begin{abstract}
Successful treatment of psychiatric disorders, including bipolar disorder and schizophrenia, is complicated and is affected by a broad range of factors associated with the diagnosis, choice of treatment and social factors. In these patients, treatment management must focus on accurate and early diagnosis, to ensure that correct treatment is administered as soon as possible. In both disorders, the treatment of the disease in the acute phase must be maintained long term to provide continuous relief and normal function; the treatment choice in the early stages of the disease may impact on long-term outcomes. In schizophrenia, treatment non-compliance is an important issue, with up to $50 \%$ of patients discontinuing treatment for reasons as diverse as efficacy failure, social barriers, and more commonly, adverse events. Treatment non-compliance also remains an issue in bipolar disorder, as tolerability of mood stabilizers, especially lithium, is not always good, and combination treatments are frequent. In order to achieve an optimal outcome in which the patient continues with their medication lifelong, treatment should be tailored to each individual, taking into account treatment and family history, and balancing efficacy with tolerability to maximize patient benefit and minimize the risk of discontinuation. These case studies illustrate how treatment should be monitored, tailored and often changed over time to meet these needs.
\end{abstract}

Keywords: bipolar disorder, recurrence, treatment management, schizophrenia, non-compliance, adverse events

\section{Introduction}

Successful treatment of psychiatric disorders, such as bipolar disorder and schizophrenia, should ideally result in long-term control in which the disease is managed effectively. However, successful treatment is complicated by a number of compounding factors, such as compliance and drug-resistance phenomena (Altamura 1990). Of these, time to accurate diagnosis is a particular issue as this can delay initiation of the correct treatment for the patient (Berk et al 2007). Administration of effective treatment is essential both in the acute phase to manage the disease rapidly, but also in the long term to ensure that treatment benefits are maintained (Altamura et al 2000). Successful treatment can be considered to be one that provides the most effective balance between efficacy and tolerability (Bowden 1995; Thomas 2007). While the initial treatment may be effective in managing acute symptoms of schizophrenia and manic or depressive episodes of bipolar disorder, continuous assessment of the patient is required to ensure optimal tolerability and efficacy (Altamura 1992). It is recognized that maintaining the initial treatment is ideal; however, alternative or additional treatments may be required at particular stages of the disease, as the course develops, to address any imbalance between efficacy and tolerability. The need for such continuous assessment and flexibility in treatment is demonstrated by the high incidence of 
treatment non-compliance in psychiatric disorders, which is a major factor in determining the outcomes of treatment (Altamura et al 2000; Dolder et al 2002; Thomas 2007). Sociodemographic factors, such as family history and employment status, are associated with poor treatment compliance, while treatment-related factors, such as lack of efficacy and adverse events and, particularly in bipolar disorder, disease symptoms (eg, poor insight and cognitive deficits) may lead directly to discontinuation of therapy (Gray et al 2002; Rettenbacher et al 2004; Linden et al 2006; Clatworthy et al 2007). The importance of adverse events is particularly true for schizophrenia, in which patients frequently receive high doses of antipsychotic drugs which affects compliance, particularly with first generation antipsychotics (Altamura 1990; Altamura et al 2000, 2007).

A further consideration is the presentation of pediatric and adolescent forms of psychiatric disorders which may differ somewhat in terms of symptoms, disease course, and treatment options from the corresponding adult disorders. This can be seen in pediatric bipolar disorder which is often characterized by the presence of mixed-mania and rapid cycling and a high degree of comorbidity with behavioral and attention disorders (Chang and Ketter 2001) - factors that contribute to difficulties in diagnosis. Similar problems are encountered in childhood onset schizophrenia which may be difficult to differentiate from affective and personality disorders due to the predominance of negative symptoms (Masi et al 2006). Treatment of early and very early-onset schizophrenia is further complicated by differences in response to pharmacological therapy compared with adults, a problem exacerbated by a lack of clinical trials in this patient sub-population (Young and Findling 2004).

This article explores some of the challenges of successfully treating patients with bipolar disorder and schizophrenia. In both of these conditions successful treatment is often hindered by misdiagnosis, poor treatment compliance and inter-patient variability in treatment efficacy and tolerability. These issues are highlighted through the use of case studies giving an insight into how to maximize clinical success in difficult-to-treat patients with diverse psychiatric disorders.

\section{Managing treatment options in bipolar disorder}

Mania is the basis for the diagnosis of bipolar disorder. Pure euphoric mania is usually easy to identify; but other types of presentation, such as psychotic mania, or when symptoms of mania and depression occur together as a mixed episode, are frequently misdiagnosed. Mixed hypomania is more frequent in patients with type I bipolar disorder and has been reported in 57\% of patients (Suppes et al 2005). Such co-occurring symptoms can make diagnosis of bipolar disorder difficult, such that there is often a delay between the onset of symptoms and correct diagnosis (Hantouche et al 2006). Inaccurate diagnosis commonly results in patients receiving incorrect therapies, which may delay successful treatment of the initial episodes, and in some cases may even exacerbate them, for example causing rapid-cycling states (Sachs et al 2007). The following case study illustrates the difficulties associated with the treatment of a patient presenting with mixed symptoms of bipolar disorder.

\section{Clinical case study}

A 36-year-old female with no previous psychiatric history was first hospitalized due to an acute episode with psychotic symptoms (reference and grandiosity delusions), high anxiety, irritability, global insomnia and agitation. She was diagnosed with brief psychotic disorder which was treated with thioridazine up to $200 \mathrm{mg} /$ day. Four months later, she suffered a major depression that was treated by a private psychiatrist with fluoxetine up to $40 \mathrm{mg} /$ day. After 1 month, the depression progressed to a mixed state with high irritability, anxiety, demanding behavior, insomnia, and depressive thoughts. Fluoxetine was stopped and the patient was treated with low doses of haloperidol (up to $5 \mathrm{mg} /$ day). During the following weeks, the patient progressively went to a new depressive episode with hypothymia (low mood), tiredness, hypersomnia, and psychomotor inhibition. Haloperidol was tapered off but major depression lasted a further 3 months. The patient visited another psychiatrist who started imipramine up to $150 \mathrm{mg}$ /day to treat the depression, this resulted in a fast improvement of the depressive episode. About 2 months later, the patient experienced another acute episode, very similar to the first one, that required hospitalization. At this time, approximately 2 years after the first episode, she was diagnosed with bipolar disorder type I, manic episode with congruent psychotic symptoms. Following the diagnosis, the patient was treated with lithium $(1000 \mathrm{mg} /$ day; serum level $0.87 \mathrm{meq} / \mathrm{L}$ ) for the following 1.5 years; however, she still experienced both depressive and hypomanic episodes of an irritable nature. Combined treatment with lithium and antidepressants (first fluoxetine up to $40 \mathrm{mg} /$ day; then imipramine up to $150 \mathrm{mg}$ /day) improved the severity of the episodes, removing psychotic tendencies, but the frequency of episodes increased to 2-week cycles of depression following by 1 week of hypomania and 1-2 weeks of euthymia. To address this rapid cycling, carbamazepine up to $600 \mathrm{mg}$ /day was added 
to the treatment regimen; however, this caused skin rashes and was discontinued after 2 weeks of treatment.

At this point, the patient first came to our clinic. Subclinical hypothyroidism was detected and was treated with thyroxine $100 \mu \mathrm{g} / \mathrm{day}$, antidepressants were discontinued and the patient was switched from lithium to sodium valproate $2000 \mathrm{mg} /$ day over a period of 3 weeks. Lithium was tapered off and valproate was started at $1000 \mathrm{mg} /$ day, resulting in serum levels of $27.1 \mu \mathrm{g} / \mathrm{mL}$. Valproate was increased to $2000 \mathrm{mg} /$ day, resulting in serum levels of $75 \mu \mathrm{g} / \mathrm{mL}$. After two milder cycles, the patient was stabilized and began to recover pre-morbid functioning.

The patient maintained this treatment regimen for 5 years during which time she experienced weight gain. Patient dissatisfaction led to the gradual replacement of valproate with lamotrigine, up to $200 \mathrm{mg} /$ day. Three months after switching the medication, the patient suffered a mixed depression with hypothymia, apathy, depressive thoughts, mood lability, irritability and insomnia. The previous regimen with valproate was restarted; the patient stabilized and was euthymic with only occasional anxiety in response to family problems. This good global functioning currently allows the patient to care for her mother, who suffers from an advanced mixed dementia, and her father. Other than weight gain, valproate was generally well tolerated and no other adverse events were experienced.

\section{Discussion}

The patient described provides a clear example of the complications surrounding successful treatment of bipolar disorder. Correct diagnosis of bipolar disorder is often delayed by over 5 years due to the presence of mixed symptoms (Hantouche et al 2006; Berk et al 2007). This prevents correct treatment from being administered and can affect the long-term outcomes of the disease (Berk et al 2007). The diagnosis of bipolar disorder type I in this patient was delayed by 2 years due to previous misdiagnoses of brief psychiatric disorder and depression, during which time the patient was mostly suffering an affective episode which resulted in two hospitalizations and complete dysfunction. Therefore, although the time of misdiagnosis was relatively short, the clinical consequences of the misdiagnosis were severe.

Mood stabilizers are the mainstay of treatment for bipolar disorder; these include lithium and sodium valproate (Bowden et al 2005, 2006). Sodium valproate has demonstrated improved efficacy over lithium in treating mixed episodes in bipolar disorder, with better outcomes reported for depressive episodes and overall function (Bowden et al
2005). Other anticonvulsants used in the treatment of bipolar disorder include carbamazepine and lamotrigine.

Rapid cycling is a common observation in bipolar disorder, and is associated with reduced treatment responses, poorer long-term prognosis and a probable higher suicide risk than patients who do not display rapid cycling (Schneck 2006). Furthermore, these patients often experience more depressive than manic episodes. As observed in this patient, subclinical hypothyroidism can be related to rapid cycling (Papadimitriou et al 2005). This finding and the substitute hormone treatment helped the patient to stabilize. Rapid cycling is estimated to occur in 14\%-53\% of patients (Maj et al 1994; Tondo and Baldessarini 1998; Suppes et al 2001), of which over $70 \%$ have been attributed to a poor response to lithium. (Dunner and Fieve 1974; Bowden 1995; Calabrese et al 2005). Longterm open clinical trials with sodium valproate in patients with rapid cycling have demonstrated initial good responses, with few recurrent episodes (McElroy et al 1988; Calabrese and Delucchi 1990). This supports suggestions that sodium valproate has a broader spectrum of use than lithium (Bowden 1995). Although the most recent data from a long-term study by Calabrese and colleagues did not support the hypothesis that sodium valproate was more effective than lithium for the treatment of rapid cycling in bipolar disorder, there was a trend towards a greater advantage with sodium valproate in terms of mood symptoms and adverse events (Calabrese et al 2005). Furthermore, sodium valproate has demonstrated antidepressive effects in bipolar disorder, preventing or delaying the onset of depressive episodes (Ghaemi and Goodwin 2001; Bowden et al 2005).

Carbamazepine has been reported to be effective in some patients with rapid cycling bipolar disorder (Post et al 1986; Joyce 1988; Tondo et al 2003), especially when used in combination with lithium (Baethge et al 2005). However, this combination caused a rash in the patient presented here and was subsequently discontinued; this is in agreement with studies showing an increase in adverse events with combined carbamazapine/lithium therapy (Baethge et al 2005). A small number of reports have demonstrated effectiveness of lamotrigine in rapid-cycling, especially when combined with valproate (da Rocha et al 2007). This was the rationale for converting the patient from valproate to lamotrigine following the onset of weight gain. However, lamotrigine monotherapy was associated with a regression of symptoms in this patient. The reintroduction of valproate improved the severity of the episodes, leading to good global functioning and stabilization, but problems with weight gain continued. Referral to a dietician and subsequent life-style changes regarding diet 
and exercise regimens may improve weight gain in patients with bipolar disorder (Chue and Kovacs 2003).

Outcomes of bipolar disorder can also be negatively influenced by incorrect administration of mood stabilizers and antidepressants (Goldberg et al 2007), and rapid cycling can be induced or worsened by antidepressants in the absence of a mood stabilizer (Sachs et al 2007). It is possible that both the use of a high dose of fluoxetine $(40 \mathrm{mg}$ /day vs the standard $20 \mathrm{mg} /$ day) and the combination of lithium and antidepressants in the patient described here exacerbated the hypomanic episodes and led to the presentation of the rapid cycling state.

Early and accurate diagnosis of bipolar disorder is essential to ensure that correct treatment is received at the earliest opportunity since incorrect treatment can negatively affect outcomes. As demonstrated in the case presented, correct treatment of bipolar disorder can be successful in the long term once this is achieved.

\section{Non-compliance and treatment choices in schizophrenia}

Treatment compliance is essential for the long-term control of schizophrenia. Despite advances in the newer atypical (second-generation) antipsychotic therapies, treatment noncompliance is reported to occur in up to $50 \%$ of patients with schizophrenia (Fenton et al 1997; Dolder et al 2002; Gray et al 2002; Rettenbacher et al 2004; Thomas 2007), with discontinuation rates of $74 \%$ within 18 months reported in some studies (Lieberman et al 2005). The impact of noncompliance is far reaching in maintenance therapy, with non-compliant patients often experiencing impaired longterm outcomes including higher relapse rates and the need for rehospitalization (Fenton et al 1997; Ascher-Svanum et al 2006). Factors associated with treatment non-compliance are multifactoral including sociodemographic variables (age, occupation, social status or level of education), attitudes of patients and carers/family towards the illness and treatment, illness-related issues such as psychopathology and comorbidities, and treatment issues such as adverse events (Rettenbacher et al 2004; Linden et al 2006). One of the most common reasons cited for non-compliance of treatment in schizophrenia are adverse events (Naber and Karow 2001). The spectrum of adverse events with antipsychotic therapy is varied and is dependent on the choice of drug. Among the most common adverse events with typical antipsychotics are extrapyramidal symptoms (EPS), sexual dysfunction, and weight gain. The newer atypical antipsychotic drugs have been associated with fewer EPS (Geddes et al 2000;
Davis et al 2003; Lieberman et al 2005); however, there have been increasing concerns that these drugs lead to metabolic side effects including diabetes, dyslipidemia, and obesity (Mortimer et al 2003; Meyer and Koro 2004; Peuskens et al 2007; Spurling et al 2007). Switching non-compliant patients to alternative antipsychotics may improve compliance by better balancing efficacy and tolerability (Peuskens 2002; Linden et al 2006; Linden et al 2007). The following case study illustrates the issues surrounding the treatment of schizophrenia, demonstrating that many changes in therapy may be required to determine the most effective treatment for any individual.

\section{Clinical case study}

A 40-year-old male presented with the appearance of persecutory delusions with sub-threshold depressive symptoms. At the time of presentation, the patient was receiving aripiprazole $15 \mathrm{mg}$ /day, which had a good impact on negative symptoms and social abilities. However, the aripiprazole was causing gastrointestinal side effects including severe nausea and constipation leading to occasional dissatisfaction and a lack of treatment compliance. The patient was also experiencing concomitant obesity with a body weight of $96 \mathrm{~kg}$ and glucose intolerance $(156 \mathrm{mg} / \mathrm{dL})$. The patient was diagnosed with paranoid schizophrenia according to DSM-IV criteria and structured clinical interview. This was episodic in nature with residual symptoms occurring between acute episodes. This diagnosis was based on the prevalence of organized delusions and hallucinations, and the separation of several acute psychotic episodes with periods of negative and depressive symptoms.

Treatment history revealed that the patient was predisposed to develop EPS and had a variable response to prior antipsychotic therapy. From the age of onset at 30 -years, the patient's initial treatment with haloperidol up to $10 \mathrm{mg}$ /day for 2 years managed positive symptoms and was used in maintenance therapy. However, the development of negative symptoms and emergence of EPS led to a change in therapy to risperidone ( $8 \mathrm{mg} /$ day). After 1 year, the risperidone dose was reduced to $4 \mathrm{mg}$ /day because of further EPS, but this resulted in the reappearance of positive symptoms and treatment was subsequently switched to olanzapine $20 \mathrm{mg} /$ day. The patient received olanzapine for 1.5 years during which time positive symptoms regressed. However, during this time the patient experienced a significant weight gain of approximately $10 \mathrm{~kg}$. This weight gain led to the patient switching treatment from olanzapine to aripiprazole up to $15 \mathrm{mg} /$ day. 
After 2 years of aripiprazole therapy, the patient presented at our clinic as described above. The patient was converted to amisulpride at a starting dose of $400 \mathrm{mg} /$ day, because of psychotic recurrences and low tolerability to other antipsychotic drugs. Amisulpride was gradually increased to an effective dose of $800 \mathrm{mg} /$ day. This approach was undertaken to avoid any risk of treating abruptly at the optimal effective dose in the acute phase of the illness given the patient's very complex medical history. There was regular monitoring of blood pressure (weekly), electrocardiogram and prolactin plasma levels (at baseline, at month 1, and every 3 months). All the parameters remained within normal levels during treatment, with the exception of prolactin which showed an increase at month $3(43 \mathrm{ng} / \mathrm{mL})$, that persisted at the other timepoints. The patient was not converted to clozapine due to the recent significant weight gain with olanzapine and his current obesity status, since the potential risk of developing a metabolic syndrome with clozapine is similar to that with olanzapine in terms of weight gain. Given that the patient had previously shown the reappearance of positive symptoms with doses $<4 \mathrm{mg} /$ day of risperidone, quetiapine was not considered useful since there was the possibility of worsening positive symptoms because of the weaker blockade of dopamine (D2) receptors.

Treatment with amisulpride led to remission of psychotic episodes with an improvement in depressive symptoms and somatizations. The patient is still being monitored and will remain on this treatment until complete remission of psychotic episodes, depressive symptoms and somatizations. Globally, the treatment with amisulpride well tolerated. There was an improvement in the patient's glucose intolerance (156 $\mathrm{mg} / \mathrm{dL}$ vs $102 \mathrm{mg} / \mathrm{dL}$ at baseline and month 6, respectively). The patient was referred to a dietology specialist in order to manage obesity, after 6 months receiving amisulpride the patient's body weight had reduced to $92 \mathrm{~kg}$.

\section{Discussion}

Treatment non-compliance has a significant impact on the outcomes of schizophrenia. Many of the currently available antipsychotic therapies have comparable efficacy in the treatment of symptoms in the acute phase; however, their tolerability profiles can vary (Davis et al 2003; Mortimer et al 2003). The presented case study demonstrates such variability, with the patient experiencing a range of complications under different therapies. This was further complicated by the emergence of different symptoms of the disease during long-term therapy. At the time of presentation, the patient was receiving aripiprazole, having already previously received therapy with haloperidol, risperidone and olanzapine. While poor compliance to aripiprazole at presentation was attributed to the gastrointestinal side effects, a large number of external factors are associated with treatment non-compliance in patients with schizophrenia, including social status, occupation and social support (Linden et al 2006). This patient was unemployed with no social support with a family history of mental health issues; his father suffered from alcohol dependence and his sister had probable schizoaffective disorder, bipolar type. These additional contributing factors to compliance should be assessed on an individual basis when considering antipsychotic therapy.

Amisulpride was considered to be the best choice of antipsychotic therapy for the patient, based on the low tolerability to other antipsychotic therapies and the presentation of depressive symptoms. Switching to amisulpride has previously been demonstrated to improve treatment compliance (Linden et al 2006; Kim et al 2007; Linden et al 2007; Spurling et al 2007). This may be related to the improved tolerability profile associated with amisulpride when comparing weight gain, metabolic control and EPS, which were particular issues for the patient illustrated here. As with most atypical antipsychotics, amisulpride is associated with a low incidence of EPS (Wetzel et al 1998; Herrera-Estrella et al 2005; Nuss et al 2007). Furthermore, clinical studies with amisulpride and olanzapine have demonstrated that patients receiving amisulpride have less weight gain and less increase in blood glucose than those receiving olanzapine (Mortimer 2004; Mortimer et al 2004; Peuskens et al 2007). Amisulpride has clear benefits over risperidone with regard to weight gain as well (Sechter et al 2002). Importantly, these studies clearly demonstrate comparable efficacy between the different agents, in agreement with an earlier meta-analysis of the efficacy of atypical antipsychotics (Davis et al 2003).

Although amisulpride has demonstrated good efficacy in the treatment of positive symptoms (Sechter et al 2002; Mortimer et al 2004; Herrera-Estrella et al 2005; Nuss et al 2007), it is also effective for the treatment of negative and depressive symptoms of schizophrenia (Speller et al 1997; Muller et al 2002; Peuskens et al 2002; Mortimer et al 2004; Herrera-Estrella et al 2005; Murphy et al 2006; Kim et al 2007; Nuss et al 2007), having clear benefits over olanzapine and risperidone with improved symptom control and longer time to depressive episodes (Peuskens et al 2002; Mortimer 2004; Kim et al 2007). However, olanzapine has also been attributed to have treatment effects on negative symptoms (Lecrubier et al 2006). 
The prolactin-elevating effects of amisulpride are well recognized, therefore prolactin plasma levels were measured regularly in this patient. In this case, no clinical symptoms of hyperprolactinemia were detected. Nevertheless, patients treated with amisulpride should be observed and asked about symptoms such as sexual dysfunction, galactorrhea and amenorrhea.

The balance between efficacy and tolerability is an essential aspect when considering antipsychotic treatment and the establishment of a positive tolerability/efficacy ratio should be an integral determinant in the choice of treatment. Whilst the efficacy of treatment is important, maintaining good long-term tolerability is essential to ensure that the treatment is continued and be of benefit. There are considerable differences in the tolerability of different antipsychotic therapies, and there may also be considerable difference in the response of different patients to these drugs. The tolerability/efficacy ratio therefore provides a measure to help to tailor antipsychotic therapy individually; it is essential that both aspects be considered when starting or switching therapies in schizophrenia. This case illustrates the benefit of continuously monitoring and changing treatment of schizophrenia to achieve this optimal treatment ratio (Altamura et al 2007).

\section{Conclusions}

Early and accurate diagnosis is essential for the successful treatment of psychiatric disorders. As well as having a beneficial impact on treatment of the acute phase of the disease, it can also affect long-term outcomes of treatment. For example, a high incidence of treatment non-compliance is reported across a range of psychiatric disorders, many of which are attributed to treatment effects such as lack of efficacy or adverse events. This is highlighted by the two cases reported here, where misdiagnosis and inappropriate drug therapy resulted in poor treatment outcomes in both bipolar disorder and schizophrenia. Although the specific factors contributing to successful treatment may vary between psychiatric disorders, the approach to treatment should be very similar. Diagnosis and treatment should be tailored to the individual patient, taking into account a detailed family and treatment history, and ensuring that the tolerability is adequate to maximize long-term compliance of medication.

\section{References}

Altamura AC. 1990. Drug-resistance phenomena in major psychoses:their discrimination and causal mechanisms. Clin Neuropharmacol, 13: S1-15.

Altamura AC. 1992. A multidimentional (pharmacokinetic and clinicalbiological) approach to neuroleptic response in Schizophrenia. Schizophrenia Res, 8:187-98.
Altamura AC, Bobes J, Cunningham D, et al. 2000. Schizophrenia: diagnosis and continuing treatment. Principles of practice from the European Expert Panel on the Contemporary Treatment of Schizophrenia. Int $J$ Psych Clin Pract, Suppl 1:1-11.

Altamura AC, Bobo VB, Meltzer HY. 2007. Factors affecting outcome in Schizophrenia and their relevance for psychopharmacological treatment. Int Clin Psychopharmacol, 22:249-67.

Ascher-Svanum H, Faries DE, Zhu B, et al. 2006. Medication adherence and long-term functional outcomes in the treatment of schizophrenia in usual care. J Clin Psychiatry, 67:453-60.

Baethge C, Baldessarini RJ, Mathiske-Schmidt K, et al. 2005. Long-term combination therapy versus monotherapy with lithium and carbamazepine in 46 bipolar I patients. J Clin Psychiatry, 66:174-82.

Berk M, Dodd S, Callaly P, et al. 2007. History of illness prior to a diagnosis of bipolar disorder or schizoaffective disorder. J Affect Disord, 23:23.

Bowden CL. 1995. Predictors of response to divalproex and lithium. J Clin Psychiatry, 56:25-30.

Bowden CL, Collins MA, McElroy SL, et al. 2005. Relationship of mania symptomatology to maintenance treatment response with divalproex, lithium, or placebo. Neuropsychopharmacology, 30:1932-9.

Bowden CL, Swann AC, Calabrese JR, et al. 2006. A randomized, placebocontrolled, multicenter study of divalproex sodium extended release in the treatment of acute mania. J Clin Psychiatry, 67:1501-10.

Calabrese JR, Delucchi GA. 1990. Spectrum of efficacy of valproate in 55 patients with rapid-cycling bipolar disorder. Am J Psychiatry, 147:431-4.

Calabrese JR, Shelton MD, Rapport DJ, et al. 2005. A 20-month, doubleblind, maintenance trial of lithium versus divalproex in rapid-cycling bipolar disorder. Am J Psychiatry, 162:2152-61.

Chang KD, Ketter TA. 2001. Special issues in the treatment of paediatric bipolar disorder. Expert Opin Pharmacother, 2:613-22.

Chue P, Kovacs CS. 2003. Safety and tolerability of atypical antipsychotics in patients with bipolar disorder: prevalence, monitoring and management. Bipolar Disord, 5:62-79.

Clatworthy J, Bowskill R, Rank T, et al. 2007. Adherence to medication in bipolar disorder: a qualitative study exploring the role of patients' beliefs about the condition and its treatment. Bipolar Disord, 9:656-64.

da Rocha FF, Soares FM, Correa H, et al. 2007. Addition of lamotrigine to valproic acid: A successful outcome in a case of rapid-cycling bipolar affective disorder. Prog Neuropsychopharmacol Biol Psychiatry, 31:1548-9. Epub 2007 Jun 22.

Davis JM, Chen N, Glick ID. 2003. A meta-analysis of the efficacy of secondgeneration antipsychotics. Arch Gen Psychiatry, 60:553-64.

Dolder CR, Lacro JP, Dunn LB, et al. 2002. Antipsychotic medication adherence: is there a difference between typical and atypical agents? Am J Psychiatry, 159:103-8.

Dunner DL, Fieve RR. 1974. Clinical factors in lithium carbonate prophylaxis failure. Arch Gen Psychiatry, 30:229-33.

Fenton WS, Blyler CR, Heinssen RK. 1997. Determinants of medication compliance in schizophrenia: empirical and clinical findings. Schizophr Bull, 23:637-51.

Geddes J, Freemantle N, Harrison P, et al. 2000. Atypical antipsychotics in the treatment of schizophrenia: systematic overview and metaregression analysis. $B M J, 321: 1371-6$.

Ghaemi SN, Goodwin FK. 2001. Long-term naturalistic treatment of depressive symptoms in bipolar illness with divalproex vs. lithium in the setting of minimal antidepressant use. J Affect Disord, 65:281-7.

Goldberg JF, Perlis RH, Ghaemi SN, et al. 2007. Adjunctive antidepressant use and symptomatic recovery among bipolar depressed patients with concomitant manic symptoms: findings from the STEP-BD. Am $J$ Psychiatry, 164:1348-55.

Gray R, Wykes T, Gournay K. 2002. From compliance to concordance:a review of the literature on interventions to enhance compliance with antipsychotic medication. J Psychiatr Ment Health Nurs, 9:277-84.

Hantouche EG, Akiskal HS, Azorin JM, et al. 2006. Clinical and psychometric characterization of depression in mixed mania: a report from the French National Cohort of 1090 manic patients. J Affect Disord, 96:225-32. 
Herrera-Estrella M, Apiquian R, Fresan A, et al. 2005. The effects of amisulpride on five dimensions of psychopathology in patients with schizophrenia: a prospective open-label study. BMC Psychiatry, 5:22.

Joyce PR. Carbamazepine in rapid cycling bipolar affective disorder. 1988. Int Clin Psychopharmacol, 3:123-9.

Kim SW, Shin IS, Kim JM, et al. 2007. Amisulpride versus risperidone in the treatment of depression in patients with schizophrenia: A randomized, open-label, controlled trial. Prog Neuropsychopharmacol Biol Psychiatry, 31:1504-9. Epub 2007 Jul 13.

Lecrubier Y, Quintin P, Bouhassira M, et al. 2006. The treatment of negative symptoms and deficit states of chronic schizophrenia: olanzapine compared to amisulpride and placebo in a 6-month double-blind controlled clinical trial. Acta Psychiatr Scand, 114:319-27.

Lieberman JA, Stroup TS, McEvoy JP, et al. 2005. Effectiveness of antipsychotic drugs in patients with chronic schizophrenia. $N$ Engl J Med, 353:1209-23. Epub 2005 Sep 19.

Linden M, Eich FX, Pyrkosch L. 2007. Do differences in atypical antipsychotics matter in routine practice? Medication switch from olanzapine and risperidone to amisulpride. Int Clin Psychopharmacol, 22:175-8.

Linden M, Scheel T, Eich FX. 2006. Improvement of patient compliance after switching from conventional neuroleptics to the atypical neuroleptic amisulpride. J Psychopharmacol, 20:815-23. Epub 2006 Jan 9.

McElroy SL, Keck PE Jr, Pope HG Jr, et al. 1988. Valproate in the treatment of rapid-cycling bipolar disorder. J Clin Psychopharmacol, 8:275-9.

Maj M, Magliano L, Pirozzi R, et al. 1994. Validity of rapid cycling as a course specifier for bipolar disorder. Am J Psychiatry, 151:1015-9.

Masi G, Mucci M, Pari C. 2006. Children with schizophrenia: clinical picture and pharmacological treatment. CNS Drugs, 20:841-66.

Meyer JM, Koro CE. 2004. The effects of antipsychotic therapy on serum lipids: a comprehensive review. Schizophr Res, 70:1-17.

Mortimer AM. 2004. How do we choose between atypical antipsychotics? The advantages of amisulpride. Int $J$ Neuropsychopharmacol, 7:S21-5.

Mortimer A, Martin S, Loo H, et al. 2004. A double-blind, randomized comparative trial of amisulpride versus olanzapine for 6 months in the treatment of schizophrenia. Int Clin Psychopharmacol, 19:63-9.

Mortimer A, Williams P, Meddis D. 2003. Impact of side-effects of atypical antipsychotics on non-compliance, relapse and cost. J Int Med Res, 31:188-96.

Muller MJ, Wetzel H, Benkert O. 2002. Differential effects of high-dose amisulpride versus flupentixol on latent dimensions of depressive and negative symptomatology in acute schizophrenia: an evaluation using confirmatory factor analysis. Int Clin Psychopharmacol, 17:249-61.

Murphy BP, Stuart AH, Wade D, et al. 2006. Efficacy of amisulpride in treating primary negative symptoms in first-episode psychosis: a pilot study. Hum Psychopharmacol, 21:511-7.

Naber D, Karow A. 2001. Good tolerability equals good results: the patient's perspective. Eur Neuropsychopharmacol, 11:S391-6.

Nuss P, Hummer M, Tessier C. 2007. The use of amisulpride in the treatment of acute psychosis. Therapeutics and Clin Management, 3:3-11.

Papadimitriou GN, Calabrese JR, Dikeos DG, et al. 2005. Rapid cycling bipolar disorder: biology and pathogenesis. Int J Neuropsychopharmacol, 8:281-92. Epub 2005 Mar 1.
Peuskens J. 2002. Switching to amisulpride. Curr Med Res Opin, 18: s23-8.

Peuskens J, De Hert M, Mortimer A. 2007. Metabolic control in patients with schizophrenia treated with amisulpride or olanzapine. Int Clin Psychopharmacol, 22:145-52.

Peuskens J, Moller HJ, Puech A. 2002. Amisulpride improves depressive symptoms in acute exacerbations of schizophrenia: comparison with haloperidol and risperidone. Eur Neuropsychopharmacol, 12:305-10.

Post R, Uhde T, Kramlinger K, et al. 1986. Carbamazepine treatment of mania: clinical and biochemical aspects. Clin Neuropharmacol, 9:547-9.

Rettenbacher MA, Burns T, Kemmler G, et al. 2004. Schizophrenia: attitudes of patients and professional carers towards the illness and antipsychotic medication. Pharmacopsychiatry, 37:103-9.

Sachs GS, Nierenberg AA, Calabrese JR, et al. 2007. Effectiveness of adjunctive antidepressant treatment for bipolar depression. $N$ Engl $J$ Med, 356:1711-22. Epub 2007 Mar 28.

Schneck CD. 2006. Treatment of rapid-cycling bipolar disorder. J Clin Psychiatry, 67:22-7.

Sechter D, Peuskens J, Fleurot O, et al. 2002. Amisulpride vs. risperidone in chronic schizophrenia: results of a 6-month double-blind study. Neuropsychopharmacology, 27:1071-81.

Speller JC, Barnes TR, Curson DA, et al. 1997. One-year, low-dose neuroleptic study of in-patients with chronic schizophrenia characterised by persistent negative symptoms. Amisulpride v. haloperidol. Br J Psychiatry, 171:564-8.

Spurling RD, Lamberti JS, Olsen D, et al. 2007. Changes in metabolic parameters with switching to aripiprazole from another secondgeneration antipsychotic: a retrospective chart review. J Clin Psychiatry, $68: 406-9$

Suppes T, Leverich GS, Keck PE, et al. 2001. The Stanley Foundation Bipolar Treatment Outcome Network II. Demographics and illness characteristics of the first 261 patients. J Affect Disord, 67:45-59.

Suppes T, Mintz J, McElroy SL, et al. 2005. Mixed hypomania in 908 patients with bipolar disorder evaluated prospectively in the Stanley Foundation Bipolar Treatment Network: a sex-specific phenomenon. Arch Gen Psychiatry, 62:1089-96.

Thomas P. 2007. The stable patient with schizophrenia - from antipsychotic effectiveness to adherence. Eur Neuropsychopharmacol, 17: S115-22.

Tondo L, Baldessarini RJ. 1998. Rapid cycling in women and men with bipolar manic-depressive disorders. Am J Psychiatry, 155:1434-6.

Tondo L, Hennen J, Baldessarini RJ. 2003. Rapid-cycling bipolar disorder: effects of long-term treatments. Acta Psychiatr Scand, 108:4-14.

Wetzel H, Grunder G, Hillert A, et al. 1998. Amisulpride versus flupentixol in schizophrenia with predominantly positive symptomatology - a double-blind controlled study comparing a selective D2-like antagonist to a mixed D1-/D2-like antagonist. The Amisulpride Study Group. Psychopharmacology (Berl), 137:223-32.

Young CM, Findling RL. 2004. Pharmacologic treatment of adolescent and child schizophrenia. Expert Rev Neurother, 4:53-60. 
